\title{
Enabling Pro-Active User-Centered Recommender Systems: An Initial Evaluation
}

\author{
Dick C.A. Bulterman, Pablo Cesar, A.J. Jansen, Hendrik Knoche, and William Seager \\ CWI: Centrum voor Wiskunde en Informatica \\ Kruislaan 413, 1098 SJ Amsterdam, The Netherlands \\ p.s.cesar@cwi.nl, dick.bulterman@cwi.nl, jack.jansen@cwi.nl \\ University College London \\ Gower St, WC1E 6 BT London, UK \\ h.knoche@cs.ucl.ac.uk,w.seager@cs.ucl.ac.uk
}

\begin{abstract}
Traditionally, an end-user has played only a passive role when viewing commercial media: he/she is expected to consume content, not interact with it. In this paper we explore advanced user interaction techniques in the home environment. One example of such advanced interaction is the creation of micro-personalized recommendations: messages that can be used as a basis for social sharing of third-party content. We describe a system for the distributed creation of micro-personal recommendations in a consumer electronics setting and show initial user testing results.
\end{abstract}

\section{Introduction}

In this paper we report on a home system that provides new interfaces for personalized content manipulation. The system allows users to construct micropersonal recommendation messages ( $\mu \mathrm{PRMs})$, while watching conventional broadcast content. All too often, commercial digital PVRs and recommender systems manages content at the box level instead of at that of the individual. We feel that this misses a significant social opportunity. Similarly, we study recommender interfaces that target parts of program content: individual points within a program that can be identified and then used as the basis for differentiated recommendation message between users in a social network. This focus on the individual and sub-content points is the motivation for our name: micro-personal recommendation messages. The $\mu \mathrm{PRMs}$ are created on a scalable class of handheld devices, ranging from mobile telephones to pen-based computers. The $\mu \mathrm{PRMs}$ can be sent as personal recommendations to friends within a users social network. Each message contains a content pointer and an optional collection of overlay text, audio and graphics that allows customization.

Our research studies new paradigms for multimedia interaction with content that is available to social groups of uses in a consumer electronics setting. Our home architecture consists of a home media server that stores content that is provided via standard broadcast channels, via peer-to-peer content sharing networks or on high-density optical disks such as DVDs or BluRay HD content. We expect that this content will be fetched on the users behalf using an intelligent recommender system, and may be post-processed in the home to allow differentiated viewing based on the individual interests of family members.

Content reaches the home via various input paths. Once the content arrives, we support two types of enduser behavior:

- personal content selection: this is basic viewing functionality that allows a user to navigate through a set of content objects (and, where appropriate, within content objects) to find and activate particular content sequences of interest. The content may be gathered by explicit user scheduling activity (such as tagging a program in an electronic program guide), by indirect user scheduling (via a recommender system) or via a $\mu \mathrm{PRM}$ received from a family or social network member.

- micro-level recommendation tagging: the explicit identification of navigation points with a content stream. The $\mu$ PRMs are saved separately from the base content and may be used for personal navigation when the viewer returns to the content 
in the future, or as the basis for a recommendation message that one user sends to another within his family or social network.

One distinguishing characteristic of this architecture is that the $\mu$ PRMs can be created and viewed using personal remote control devices, each of which operate in parallel with the shared TV screen. These personal remote control devices form the basis of a system that supports differentiated content delivery and differentiated personal recommendation delivery and generation. The devices range from a conventional remote control, through low-powered handheld devices like telephones and minimal pen-based devices such as the Nokia N770, up to full-featured (but reduced size) tablets such as the Samsung Q1. The key question we wanted to understand is: can multiple remote controls facilitate more active interaction in a daily-use home setting?

This paper is structured as follows. Section 2 illustrates a typical use-case scenario. Section 3 provides a technical description of our client architecture. Section 4 provides a summary of results obtained from initial user testing by 12 groups of three users who were interacting in a social setting. Section 5 provides a brief summary of related work. We conclude in Section 6 with a summary of our findings and our directions for future work.

\section{Use Scenario}

Most broadcast TV consists of linear video presentations. There is typically no support for richer navigation within a programs content. An exception to this model is the scene navigation interface provided with most commercial DVDs. Here, a movie is partitioned into scenes, and a form of direct navigation interface is provided to go from one high-level scene to another. The navigation structure is not automatically deduced from content, but is separately produced.

Commercial scene-selection interfaces have several frustrating characteristics: first, content browsing can not be combined with content viewing (as an overlay, or otherwise): a DVD system is either in viewing mode or menu mode; second (and somewhat related), supplemental content (PIP, teletext) is displayed on the main screen, replacing any content already in view; third, all viewers see the same sets of selections (there is no micro-personalization of content). Personalized solutions to these issues are addressed by our environment.

The main uses of the personalized remote controls considered in this article are user-centered navigation and personalized content chaptering. If only a single conventional remote is available, all user system interaction is done via the device and the TV screen. If higher-level devices are available, a view similar to that shown in Figure 1 is presented to the devices user. Here, we see that this user has various TV control functions available at left, plus is shown two high-level content posters: one for a collection of nature programs and one for a collection of movie trailers. We use the TV-Anytime Phase-II packaging specification as a basis for content collection.

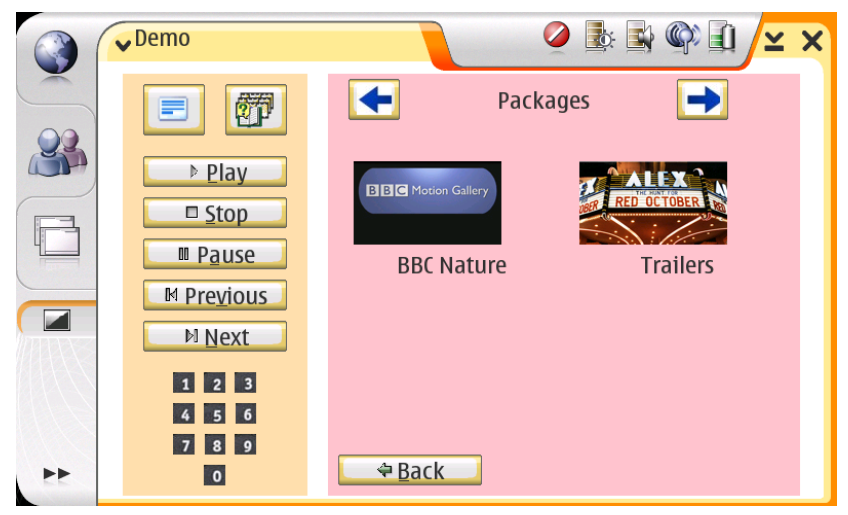

Figure 1. Personal Remote Control. Nokia N770 Interface

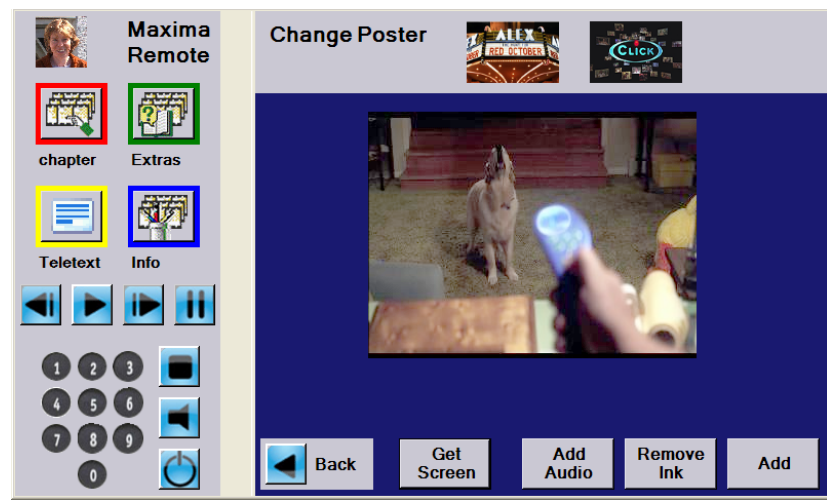

Figure 2. Creating a new recommendation poster within a program. Samsung Q1 interface

Our content hierarchy allows navigation/recommendation points to be described at three levels: the package level, where collections of programs are stored and grouped by package name; the program level, where (within packages) individual programs are identified; and the fragment level, where 
individual navigation points are identified within a program. It is possible (and a long-term interest) to have the partitioning of content between packages, programs, and fragments occur automatically. However, at every level, a user should be able to exert a personal influence over content scheduling. Content navigation and selection is supported by direct manipulation on each of the personal remotes. The user of a remote can perform functions such as checking recommendations, reading teletext or recommending content to other users while not disturbing the content flow on the main screen. More importantly, the user can also create a new navigation or recommendation points at any time while watch content by simply selecting a 'Chapter' function in the upper left hand side of the display. This brings up an interface (shown as implemented on the Samsung Q1 in Figure 2) that allows a poster image to be selected which is added to the collection of posters for that program based on its temporal positioning in the content. The viewer may add optional text captions, voice-over descriptions explaining why the recommendation point was chosen and even line-art overlays. This kind of advanced functionality may seem exaggerated for couch-top use, but is a natural extension of a personal interface. It is, of course, not required.

The final major activity provided to a user is the ability to forward $\mu$ PRMs to members of a family and friends peer group. The $\mu$ PRMs contain a copy of the poster, a description of the package/program, the address of one or more recipients and a CRID: a TVAnytime content identifier. (For private content, such as self-produced home movies or Web-stored content, the CRID is a pointer to the content.) It is important to underscore that a $\mu \mathrm{PRM}$ contains a content pointer, and not content itself. In the case of an optical disk, the recommendation message will only be useful if the recipient has a (hopefully legal!) copy of the content.

This scenario highlights the major contributions of our work:

1. The development of a personal remote control model that allows users to manipulate view metainformation and preview content in a personal setting, providing a private space in a socially-crowded living-room.

2. An interface that supports personal navigation and selection of content.

3. An interface that supports the direct recommendations of content to others in a social network. The recommendation is not limited to a particular package, but it could be target a program or a specific fragment.

\section{Client Architecture and Implementa- tion}

The client architecture within our home environment contains a collection of devices that are used to control, transmit and view media. Each client allows the personalized management of content within a home environment. The functionality provided by each client device will vary with its complexity and richness, but each of the devices allows for content selection and most non-trivial devices allow for direct navigation and recommendation management. The basic flow of control for the applications considered in this paper were presented in the Introduction. We highlight basic facilities in the paragraphs below. We consider the interface implemented on a Samsung Q1.

Figure 3 shows the left-side general control area of a device. These control (which clearly have not been subject to any industrial design scrutiny) allow content to be selected for viewing and control on the local device. The following types of viewing is support:

- Content interrogation: metadata on the current program can be displayed on the local display, allowing personal interrogation without bothering others.

- Teletext viewing: teletext can be displayed on the local display, allowing personal browsing without endangering family relationships.

- Application control: various applications allow incontent control. This is triggered with the EXTRAS button.

- General content control: main screen content can be selected and controlled via a conventional multi-button interface.

- Chaptering control: this is the ability to insert personal navigation points. More on this below.

The look and feel for these buttons for these functions will vary by control device. Note that primary advanced control functions map to the red/green/yellow/blue buttons on a conventional remote control.

The right side of a client contains an area for direct manipulation of navigation and recommendation activity. A detailed description of these functions is beyond the scope of this paper, but the general features of the gesture-based pen interface is shown in Figure 4. The key concept illustrate here is that for each package, program or collection of fragments, a user can perform a wealth of control operations that are managed in isolation for content that is being displayed on the main 


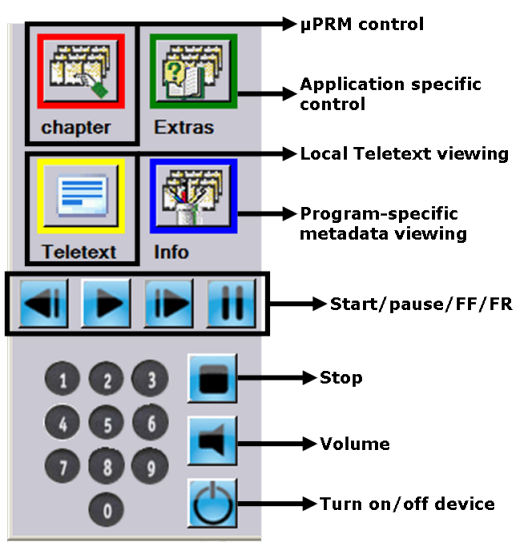

Figure 3. Left-side general control area of a personal remote control device.

screen. The content on the main screen can always be mapped to that on the local device, but the control operations do not depend directly on the main display state. Among other benefits, this makes control migration to remote devices that are not co-located with the main display screen possible.

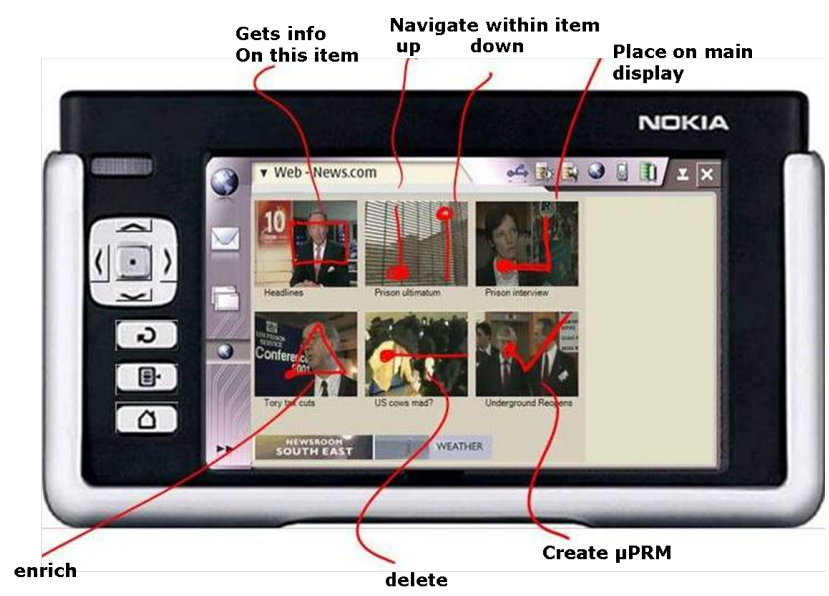

Figure 4. Gesture based interface actions.

Content navigation is performed based on the set of posters that have been defined for a particular program. These posters may be defined by the content owner (in this case, the BBC), or they may be defined by an intermediary recommendation system. The may also be defined directly by the user. When the user creates a program recommendation, a poster is taken from the active main display at the moment the leftside chaptering control button is pressed. This will result in a new navigation point being created for that program. The user may enrich the poster with additional information, including line art, a caption or an audio voice-over.

\section{$4 \quad$ User testing}

The personal chaptering system provides a collection of technology that, for some, is seen as a solution that is looking for a problem. Put another way, is it reasonable to expect that a broad user community of relatively passive users will want to manipulate TV content instead of simply viewing it? In order to answer this question, we submitted our system to user testing. This testing was done in isolation of the development.

\subsection{Method}

We used scenarios to elicit feedback on the current design of the system. The design and implementation was clearly in prototype status. We modeled a representative user community by constructing twelve groups of friends with up to three people to participate as paid subjects in the study. A total of 27 people took part in sessions that lasted for 90 minutes and were audio and video recorded. A viewing environment was developed consisting of a prototype server, three hand-held control devices (the activators) and a small library of content. Each activator had a prearranged set of packages available on them. The content that was available to the group members was overlapping but some content was available to some group members but not all of them. Each activator had at least one recommended content item on them. The shared display was a $5016: 9$ screen.

\subsection{Procedure}

After having described the procedure of the session we gave each participant of the group an activator. We suggested that these would be their personal devices that they could carry around with them. For the Nokia 770 we told them that this could also be used as a mobile phone. We asked them to imagine the use of the system in everyday situations.

We encouraged them to explore the system during the scenarios and to comment on the features or any problems they ran into at any time. Each scenario was preceded by walking the participants through the necessary functionality on the device.

Finally we administered questionnaires which collected individual preferences of the prototype's existing and potential features. 


\subsection{Results}

The user groups provided a rich and detailed set of comments on many aspects of the $\mu \mathrm{PRM}$ system. The results on the participants' preferences for the available and potential features is shown in Figure 5. In the paragraphs below, we concentrate on aspects of the results that specifically related to the generation and use of micro-personal recommendation messages. (For ease of discussion, these were referred to as chaptering messages during the study.)

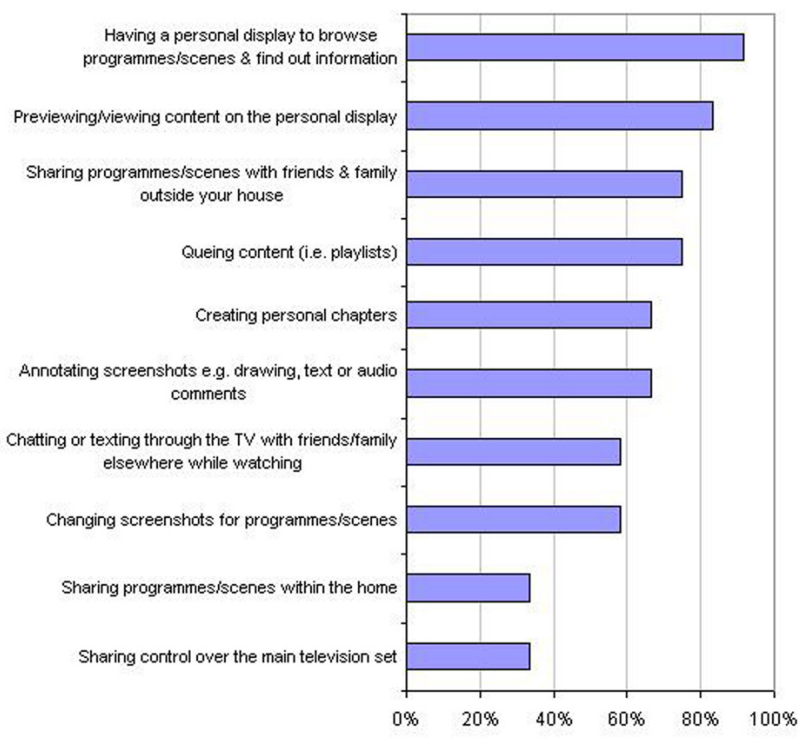

\section{Figure 5. Percentage of participants inter- ested in existing and potentials features of the prototype.}

The following initial conclusions came out of the user study:

Creating $\mu$ PRM messages: most users liked the idea of having $\mu \mathrm{PRM}$ functionality, either for creating personal bookmarks or for defining personal scene selection points. Some users wanted to define not only a single point, but also a range of content that could be recommended as a whole sub-item.

Sending and receiving $\mu P R M$ messages: Participants were generally very enthusiastic about the idea of sharing content. 'I think that would be really cool'... 'I think people would really get into that'... 'check out this trailer we've got to go and see this movie'. For the most part, they viewed this as something they would like to do with friends or family who did not live with them although they could also see the value of sending content to people living in the same household if they were in another room or else not currently present in the household. There was a desire for an improved $\mu \mathrm{PRM}$ notification and archiving system.

Benefits of a personal preview display: For many participants, a key advantage of the system was the fact that they were able to browse content and choose programmes without interrupting what was playing on the main screen. Thus, in a group viewing situation, everyone could browse for themselves without interrupting others. However, this aspect did not appeal to everyone. A number of participants did not want to browse while something was already showing. They preferred to wait until the current clip finished before they browsed for next programme.

Single versus multiple remote controls: Almost all participant groups felt that conflict would arise from multiple devices having the capacity to control a single shared display. 'If you introduce two or more remote controls, it's going to be havoc'. In particular, they envisaged children competing to control the shared display. In general, it was argued that, if that were multiple control devices, there should be some kind of hierarchical system. One suggestion was that there should be a master control. Others could have access to the shared display but only if the person owning the master control allowed shared control. Thus, a parent could allow his or her children to control the screen but could take away control if necessary. Or a host could allow visitors control. Another suggestion was the idea of passing control between devices. With a gesture, the person with the control could pass control on to someone else in the room. Alternatively, there could be a kind of jukebox system where each individual chose content to play; the content would be queued so that no one could interfere with what was currently playing. A further suggestion was that, instead of playing content, each person was able to put forward suggestions that had to be agreed on by other people present in the room.

Interestingly, nearly all groups simply used the system (with its imperfections) in a social TV viewing context as a natural extension of the social viewing paradigm.

In summary, having a personal display that allowed for browsing, personalizing and annotating content as well as sending and receiving $\mu$ PRM messages appealed to the majority of the participants. Sharing within the home did not have a large appeal to the groups of friends but this might be different for families and in shared households. We can see the desire to reciprocate recommendations as a potentially strong driver 
for the adoption of impromptu recommendations from the couch.

\section{Related Work}

Media content management is an area that has been widely studied in the past, albeit from a slightly different context than the work reported in this paper. This section provides a overview of recent work related to the topic. Much of the research on content selection within an IDTV environment has focused on the macro-level concerns of selecting an entire program among a wide range of content available to a user. This is often done by some form of recommender system [5]. While we agree that recommender systems will play an important role in the future, they provide little assistance in navigating through content once it arrives in the home.

Macro-level content selection is supported by the TV-Anytime Forum. Research in this area includes the UP-TV project [2], which presents a program guide that can be controlled and managed (e.g., delete programs) from personal handheld devices. Our work also studies navigation using personal devices, but focuses on a finer level of granularity: how fragments within a program can be managed and personalized, and then controlled using a variety of light-weight end-user devices.

The development of an automatic micro-level recommender has not been a focus of the work reported in this paper. Instead, we have focused on a content tagging and selection interface that allows an end-user to effectively scan a large content set and then provide a personalized version of that content for members of his/her peer group. Our interest has also been in defining a scalable control model that allows content-based selection to be exploited on a wide range of dissimilar control devices (from standard remote controls to tablet PCs). Neither of these topics has been broadly considered within the existing literature of IDTV systems.

We see peer group content sharing as a postmanipulation distribution activity, where the content is shared across a social network. The most relevant technology in this case is the use of P2P networks [6]. Current sharing research focuses on social activities while consuming digital media [4]. Academic prototypes include Telebuddies [3] and ConnectTV. Industrial prototypes include AmigoTV [1], Together TV, and Cose. All these systems focus on connecting television watchers by chat or voice messages. The contribution of this paper goes one step further, by allowing the viewers to make personal recommendations of content fragments, select an appropriate screenshot as an icon, enrich the icon, and share it.

\section{Conclusion}

This paper investigates advanced user interaction in a home setting. The results presented in this paper represent a first attempt to understand if this kind of functionality will be used by passive television users. The encouraging first results presented in this paper justifies further work on the deployment of a full working system, to investigate the effects of multiple extended remote controllers in a household, to compare $\mu \mathrm{PRMs}$ with other alternatives for sharing videos, and to further investigate user interface models for the public (TV) and private (extended remote control) settings.

\section{$7 \quad$ Acknowledgments}

This work is supported by the NWO Bsik project PDC3: BRICKS, and the IST-FP6 project SPICE. The development of Ambulant is supported by NLnet. UCL would like to thank the IST-2005-27034 UNIC project.

\section{References}

[1] T. Coppens, L. Trappeniers, and M. Godon. AmigoTV: towards a social TV experience. In Proceedings of EuroITV, 2004.

[2] A. Karanastasi, F. G. Kazasis, and S. Christodoulakis. A natural language model for managing TV-anytime information in mobile environments. Personal and Ubiquitous Computing, 9(5):262-272, 2005.

[3] K. Luyten, K. Thys, S. Huypens, and K. Connix. Telebuddies: social stitching with interactive television. In CHI '06: Proceedings of the SIGCHI conference on $\mathrm{Hu}$ man Factors in computing systems, pages 1049-1054, 2006.

[4] L. Oehlberg, N. Ducheneaut, J. Thornton, R. Moore, and E. Nickell. Social TV: Designing for distributed, sociable television viewing. In Proceedings of EuroITV, pages 251-259, 2006.

[5] B. Smyth and P. Cotter. A personalized television listings service. Commun. ACM, 43(8):107-111, 2000.

[6] J. Wang, J. Pouwelse, J. Fokker, and M. Reinders. Personalization of a peer-to-peer television system. In Proceedings of EuroITV, pages 147-155, 2006. 